A. Dodge et al., C.R. Physique 5 (2004),557-563.

\title{
An example of a chaotic micromixer: the cross-channel micromixer
}

\author{
Arash Dodge $^{\mathrm{a}}$, Marie-Caroline Jullien ${ }^{\mathrm{b}}, \mathrm{Yi}-\mathrm{Kuen} \mathrm{Lee}^{\mathrm{c}}, \mathrm{X} . \mathrm{Niu}^{\mathrm{c}}$, Fridolin Okkels ${ }^{\mathrm{a}}$, \\ Patrick Tabeling ${ }^{\mathrm{a}}$ \\ ${ }^{a}$ MMN, ESPCI, 10, rue Vauquelin, 75005 Paris, France \\ ${ }^{\mathrm{b}}$ SATIE/BIOMIS, ENS-Cachan, campus de Ker Lann, 35170 Bruz, France \\ ${ }^{c}$ Department of Mechanical Engineering, Hong Kong Univ. of Science and Technology \\ Hong Kong, People’s Republic of China
}

\begin{abstract}
In this article dedicated to micromixing, we concentrate here on a particular micromixer - the 'cross-channel micromixer'. This mixer exploits an oscillatory perturbation to induce chaotic trajectories, favoring mixing. We present here theory, numerical simulations and experiments performed on this system.
\end{abstract}

\section{Résumé}

Un micromélangeur chaotique : le micromélangeur en croix. Dans cet article consacré au micromélange, après une brèverevue sur le sujet, nous nous concentrons sur la présentation d'un micro-mélangeur particulier - le « micromélangeur en croix ». Il s'agit d'un système ouvert, où un écoulement stationnaire, dans un microcanal, est soumis à une perturbation hydrodynamique oscillante dans le temps ; ce système induit naturellement des trajectoires chaotiques, favorisant ainsi le mélange. L'article expose le principe de fonctionnement de ce système particulier, décrit des simulations numériques et présente une realization expérimentale.

Keywords: Micromixer; Microchannel; Chaotic mixing; KAM curves; Particles sorting Mots-clés: Micromélangeur ; Microcanal ; Mélange chaotique ; Courbes de KAM; Tri de particules 
A. Dodge et al., C.R. Physique 5 (2004), 557-563.

\section{Introduction}

Microfluidic systems are too small to shelter turbulence; thus, unlike ordinary size systems, mixing cannot bear on hydrodynamic fluctuations in microsystems. Mixing cannot rely on molecular diffusion either, because microsystems are, in many cases, too large for diffusive mixing to be viewed as a fast process. To summarize the situation, one may say that microsystems are often inhospitable to mixing. In a standard lab on a chip device, if nothing is done, it would take hundreds of seconds to develop sequences of reactions, and this is barely compatible with the general expectation that labs-on-a chip provide fast responses to analytical tasks.

These issues were raised in the early 1990s. Since then, more than twenty micromixers have been reported in the literature. This work is reviewed in [1,2]. The first micromixers consisted in dividing channels into small branches, or small nozzles, so as to enhance concentration gradients, and therefore increase diffusive exchanges. This approach has been pushed to an extreme by Knight et al. [3], who obtained mixing times on the order of tens of $\mu \mathrm{s}$. More recent achievements consist in exploiting Taylor-Aris dispersion [4], use viscoelastic properties [5], or produce recirculating flows by means of electrokinetic forces $[6,7]$, to mention a few. We will not review these approaches here. All these micromixers have been shown to 'work' - i.e., to mix efficiently, compared to pure diffusion - in some range of experimental conditions; they are usually associated to corteges of constraints and limitations, often uneasy to reconcile with integration prospectives or to adapt to material requirements. This probably explains why, over the past decade, so many microdevices have appeared in the literature. The subject is still open, since we do not have, at the moment, a universal micromixer, which would satisfy all the requirements imposed by microsystem technology and which would fit to all sort of applications microfluidic systems are dedicated to. In this respect, the situation is comparable to the one existing in the macroworld. 
A. Dodge et al., C.R. Physique 5 (2004), 557-563.

In the present paper, we focus on a particular type of micromixer, which attempts to exploit chaotic trajectories. These systems have been proposed in the last five years. They are based on the idea that chaos is remarkably efficient at mixing low Reynolds number flows. In essence, the efficiency relies on the fact that, in chaotic regimes, diffusive fluxes across interfaces are enhanced at exponential rates, in time. The idea is not new, since the domain of chaotic mixing, or laminar mixing has been developed, at a remarkable level of sophistication, in the 1980s [8]. In this respect, it is fair to say that microsystems offer a new field of application to these ideas.

The first design of a chaotic micromixer was published by Evans et al., in 1997 [9]. It consisted in a time-dependent source-sink flow, activated thermally, and attempted to adapt a system originally proposed by Aref [10]. A few years later, Liu et al. [11] (see also [12]) worked at producing a $3 \mathrm{D}$, spiraling chaotic mixer, mimicking the $\mathrm{ABC}$ flow configuration, for which it is known that chaotic trajectories exist [13]. In the same period, Volpert et al. [14] (inspired by the theoretical work of [15]) and Lee et al. [16,17] realized cross-channel micromixers, consisting of one or several channel intersections, operating by using an external oscillatory flow excitation. More recently, Stroock et al. [18] exploited threedimensional vortical flows to produce chaotic regimes along a grooved channel.

In this special series of Compte Rendus, the present article is dedicated to micromixing. The presentation concentrates on a particular system, the cross-channel micromixer; the geometry of this system is well adapted to standard microfabrication technology, and it turns out that, in the domain of chaotic mixing - well-developed in the 1990s - it defines an unusual configuration. The configuration is unusual in the sense that the flow is open, and the chaotic regimes are transient. Such systems have not received, so far, as much attention than closed systems. Microfluidics thus naturally leads to consider new configurations, and this may give opportunities to uncover novel phenomena. It happens that the cross-flow 
A. Dodge et al., C.R. Physique 5 (2004), 557-563.

micromixer is the host of a new phenomenon - a spatio-temporal resonance effect - which, to the best of our knowledge, has not been noticed before. This illustrates the idea that, by naturally considering new flow geometries, microfluidics is likely to expand our knowledge on chaotic systems.

The paper is organized as follows: we first make a general presentation of the crosschannel mixer. We then describe an experiment, where various regimes (chaotic, nonchaotic, resonant, wavy,...) were observed. We finally present a numerical analysis, using the tools of Hamiltonian dynamics to characterize the system. We conclude by suggesting a practical use which may be made for a particular regime found in this system, called 'resonance'.

\section{Experimental study of the cross-channel micromixer}

\subsection{How the cross-channel micromixer operates}

As announced in the introduction, the cross-channel micromixer has a simple geometry, which can be easily produced by using standard microfabrication techniques: it consists of a main channel where two streams of miscible liquids flow side by side, as represented schematically on Fig. 1 (first figure). The main flow is perturbed by a transverse, oscillating periodic flow driven along the side channels, across the intersection. In Fig. 1, one has a situation where the deformation of a material line, separating the two streams, and produced by the perturbation, involves stretching and folding, in a way comparable to the tendril-whorl problem. The mechanism is the following: as the material line penetrates into the intersection, it gets distorted by the action of the transverse flow; when the transverse flow stops (last figure) under certain conditions, the interface may be folded, because of the presence of velocity gradients in the main stream. Thus a tendril-whorl may be produced, as in ordinary chaotic systems, and this is favorable to mixing. The mechanism is induced by the particular topology of the streamlines, which involves two transient saddle points, located right at the 
A. Dodge et al., C.R. Physique 5 (2004), 557-563.

corners of the intersection. In order to get chaotic-like regimes, the interface must reach the vicinity of these particular points, and therefore the perturbation must have a substantial amplitude. At smaller amplitude of the perturbation, the interface does not fold, and the interface generates (unfolded) waves.

\subsection{Experimental set-up dedicated to the study of the cross-channel micromixer}

Here, we present the device we used for studying the cross-channel micromixer. The system was microfabricated in PDMS, using two-level soft lithography technology [20]. The layout of the device with its operation mode is illustrated in Fig. 3. It consists of a main channel where two streams of glycerol flow side by side, one flow being marked with fluorescein and the other not. The main flow is perturbed by a transverse, oscillating periodic flow created at the cross-channel intersection. The oscillating flow is produced by a pair of ten integrated PDMS valves displayed in a comb-shaped fashion. They were fabricated as described in [20]. The basic functioning of one of the valves is explained in Fig. 2. A waterfilled actuation channel compresses a membrane, closing or opening a segment of what is called here a fluidic channel. The membrane deformation will create a liquid displacement in the fluidic channel, with a displacement amplitude depending on the applied air pressure and the valve surface area. By periodically switching the two combs, one creates an oscillating perturbation at the cross-channel intersection. The frequency of the oscillation depends on the speed at which the two combs are switched. With this method, control of the perturbation has been found excellent for both amplitude and frequency, a feature unattainable in previous systems.

\subsection{Experimental observations}


A. Dodge et al., C.R. Physique 5 (2004), 557-563.

The fluids we use in the experiments are mixtures or $90 \%$ glycerol and $10 \%$ water, one being labelled with fluorescein, the other not. In such conditions, we reduce blurring, caused by molecular diffusion, at the interface between the labelled and unlabelled fluid, and thus, we more easily visualize the details of the patterns. Figs. 4 and 5 show two states of flow, obtained for two different values of the frequency and amplitude of the time periodic actuation, exerted on the valves. As suggested by these figures, wavy regimes are obtained at large frequencies and small amplitudes of the transverse flow, while whorl-tendrils are obtained, typically, in the opposite case. The behavior of the system at large frequencies may be explained by noting that, at fixed actuation pressure, the displacement of the material lines, in the flow, is reduced as the frequency is increased.

In the whorl-tendril regime (i.e., when the flow at the intersection folds and reorients the interface), one may expect substantial mixing to be achieved. This seems evident in Fig. 5, for the particular fluid viscosity we used. It is important to note that, after the fluid particles reach the outlet of the intersection, the system undergoes the effect of Taylor Aris dispersion: this phenomenon is due to the presence of velocity gradients, present in the main stream. This dispersion process, is an additional effect, which indeed complicates the analysis of the system; nonetheless, from the viewpoint of mixing, it is a valuable phenomenon, since it contributes to the formation of homogeneous mixtures at the outlet of the intersection.

We also observed particular states, linked to a spatio-temporal resonance effect, described in [21]. In this regime, the interface is strongly distorted at the intersection, and leaves it almost as flat as it entered. The resonant regimes appear for particular values of the frequency and the amplitude of the time periodic forcing: kinematically, they correspond to fully reversible deformations of the interface. An example of a resonant state is shown in Fig. 6. The observation is in good qualitative agreement with the numerical simulations of [21]. To the best of our knowledge, spatio-temporal resonances have not been observed in other 
A. Dodge et al., C.R. Physique 5 (2004), 557-563.

systems so far.

\section{Numerical simulations of the cross-channel mixer}

\subsection{Using Poincaré mappings to analyze the system}

Because the Reynolds number is small, the flow can be represented by Stokes equations, and the velocity field at the intersection of the main and side channels is a linear superposition of two contributions: one corresponding to the main stream and the other to the oscillating transverse flow. The details of the mathematical model is reported in [19]. The existence of chaotic regimes is well identified on Poincaré sections. There are different ways to define a Poincaré section. Four our system, Niu and Lee [19] defined it in the following way:

- First, one must replicate the X-mixer, so as to work with a spatially periodic system. This is shown in Fig. 6.

- Then for a particular trajectory, we define the set of points $P_{1}, P_{2}, \ldots, P_{n}$, representing the intersection between the lines $x_{n}=n \times p c h, n=1,2, \ldots$, and the trajectory. The position of this point depends on coordinate $y$; however, in the spirit of dynamical systems, time $t$ should also be treated as a (generalized) coordinate.

Thus obtained, the positions of $P_{n}$ should be generally thought as depending on $y_{n}$ and $t_{n}-$ the time at which the $n$th intersection occurs. For practical reasons, instead of time, we use the following variable:

$$
\alpha_{n}=\operatorname{modular}(\omega t, 2 \pi)
$$

which varies between 0 and $2 \pi$.

Fig. 9 sketches the connection between the Poincaré mapping and the physical space. 
A. Dodge et al., C.R. Physique 5 (2004), 557-563.

If the trajectory is periodic or quasi-periodic, the Poincaré sections are circles or lines, while, if the flow is chaotic, the Poincaré sections fill the available space. Fig. 8 depicts Poincare sections of the chaotic mixer for different amplitudes of perturbation. In these two figures, the dotted areas are the chaotic regions, and the circles and lines denote quasiperiodic motions or tori; particles located on tori have a periodic or quasi-periodic trajectory. The curves that separate the periodic from the chaotic areas are KAM curves [8]; such lines represent barriers to mixing.

When $v_{p}=0$, the Poincaré sections have parallel straight lines, signalling all trajectories are spatially quasi-periodic. As $v_{p}$ increases, the straight lines undergo undulations, while some of them break up into closed loops or chaotic areas. In Fig. 8(a), for $v_{p}=0.15$, a large part of the trajectories are quasi-periodic. Only trajectories, emitted from points located close to the channel walls are chaotic. As the perturbation amplitude increases, the outer KAM curves break up; the large periodic domain, gradually shrinks, giving rise to a chain of periodic islands. As $v_{p}$ increases further, the islands break again into smaller islands until all merge into a chaotic areas. When $v_{p}$ reaches 1 , chaos fills all space.

A way to identify chaotic regimes (on more quantitative grounds), is using Lyapunov Exponent (LE), usually denoted by $\lambda$. In a chaotic regime, two nearby orbits in the phase space separate exponentially with time. The Lyapunov Exponent is a measure of the average exponential rate of divergence of two such trajectories. In [19], it has been shown that, by increasing the amplitude of the forcing, for a given frequency, $\lambda$ becomes greater than 1 , revealing the existence of chaotic regimes. The study of the evolution of $\lambda$ with the control parameters of the system can thus be used to improve mixing, assuming the larger the Lyapunov exponents, the better the mixing (the link between the two notions is discussed in Ottino's textbook [8]). 
A. Dodge et al., C.R. Physique 5 (2004), 557-563.

\section{Conclusions}

To conclude, we have presented herein a particular mixer, for which a main channel stream is subjected to the effect of a transverse, time-periodic flow, at a cross-channel intersection. Simulations show that, in a system with periodic boundary conditions, there exists a range of parameters in which quasi-periodic and chaotic regimes coexist. When mixing is sought, it is preferable to work in a fully chaotic regime. Experimentally, working with a single intersection, leads to generate transient or finite-time chaotic regimes. Still, we noted, in the present article, and with the pair of fluids we considered, that one unit may be sufficient to achieve acceptable mixing.

The experiment also revealed the existence of a spatio-temporal resonance phenomena, in which material lines are elongated and folded as they penetrate in the intersection, but return to their initial shape as they get out of it. This regime is not interesting for mixing; however, it may have interesting implications for particle sorting by increasing, temporarily, the interface between the two fluids. Since the diffusion coefficient of a particle is inversely proportional to its radius, two populations of particles, with different radii, located on the same side of an interface separating two miscible fluids, will undergo a demixing process: the smaller particles will diffuse more easily across the interface than the larger ones. In such a regime, the system thus produces demixing, while, in other experimental conditions, as mentioned in this article, it produces efficient mixing. This emphasizes on the versatility of such a system, a characteristic quite remarkable, and which could be achieved, rather naturally, by using microsystem technology. This is perhaps worthwhile to mention, in a special issue of Comptes Rendus, dedicated to microfluidic systems. 
A. Dodge et al., C.R. Physique 5 (2004), 557-563.

\section{Acknowledgements}

This work was supported by Centre National de la Recherche Scientifique, ESPCI and Hong Kong University of Science and Technology under PROCORE grant (F-HK17/02T) and I2MS grant (HKUST I2MS01/02.EG08). 
A. Dodge et al., C.R. Physique 5 (2004), 557-563.

\section{References}

[1] D. Reyes, D. Iossifidis, P.-A. Auroux, A. Manz, Anal. Chem. 74 (2002) 2623; P.-A. Auroux, D. Iossifids, D. Reyes, A. Manz, Anal. Chem. 74 (2002) 2637.

[2] P. Tabeling, Une introduction à la microfluidique, in: Coll. Echelles, BELIN, 2003.

[3] J. Knight, A. Vishwanath, J. Brody, R. Austin, Phys. Rev. Lett. 80 (1998) 3863.

[4] A. Scherer, S. Quake, Science 290 (2000) 1536.

[5] A. Groissman, V. Steinberg, Nature 410 (2001) 905.

[6] A. Ajdari, Phys. Rev. E 53 (1996) 4996;

A. Ajdari, Phys. Rev. E 61 (2000) 45.

[7] J. Deval, P. Tabeling, C.M. Ho, in: Proc. MEMS, Las Vegas, USA, 2002.

[8] J.M. Ottino, The Kinematics of Mixing, Stretching, Chaos and Transport, Cambridge University Press, 1989.

[9] D. Evans, D. Liepmann, A.P. Pisano, in: Proc. MEMS 97, Nagoya, Japan, 1997, p. 96.

[10] H. Aref, S. Jones, Phys. Fluids 1 (1988) 470.

[11] R. Liu, M. Stremler, K. Sharp, M. Olsen, J. Santiago, R. Adrian, H. Aref, D. Beebe, J. Micro. Systems 9 (2) (2000) 190.

[12] D. Beebe, G. Mensing, G. Walker, Ann. Rev. Biomed. Engrg. 4 (2002) 261.

[13] T. Dombre, U. Frisch, J.M. Greene, M. Henon, A. Mehr, A.M. Soward, J. Fluid. Mech. 167 (1986) 353.

[14] M. Volpert, I. Mezic, C. Meinhart, M. Dahleh, in: Proc. ASME Mech. Engrg. Int. Congress and Exposition, Nashville, TN, 1999, p. 483.

[15] D. D’Alessandro, M. Dahleh, I. Mezic, IEEE Trans. Automat. Control 44 (1999) 1852.

[16] Y.-K. Lee, C. Shih, P. Tabeling, C.M. Ho, Characterization of mixing process in a microchannel flow, presented at the 52th Annual Meeting of APS, New Orleans, November, 1999. 
A. Dodge et al., C.R. Physique 5 (2004), 557-563.

[17] Y.-K. Lee, J. Deval, P. Tabeling, C.M. Ho, in: Proc. MEMS 2001, Interlaken, 2001, p. 483.

[18] A. Strooke, A. Ajdari, H. Stone, G. Whitesides, Science 295 (2002) 647-651.

[19] X. Niu, Y.-K. Lee, J. Micromech. Microeng. 13 (2003) 454.

[20] M. Unger, H. Chou, T. Thorsen, A. Sherer, S. Quake, Science 288 (2000) 113.

[21] F. Okkels, P. Tabeling, Phys. Rev. Lett. 92 (2003) 3. 
A. Dodge et al., C.R. Physique 5 (2004), 557-563.
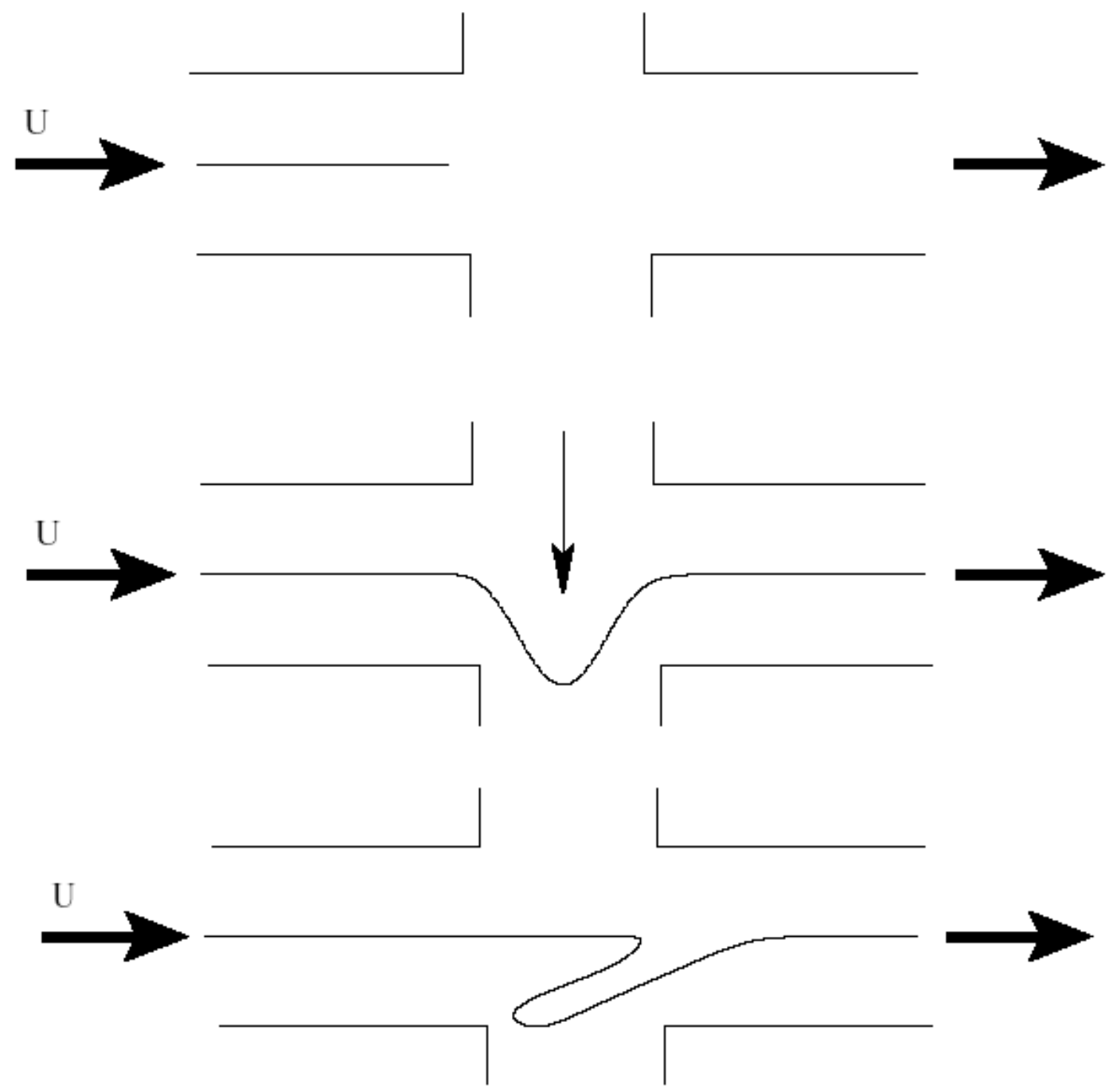

Fig. 1. Stretching and folding of an incoming material line, as takes place in the X-mixer. 


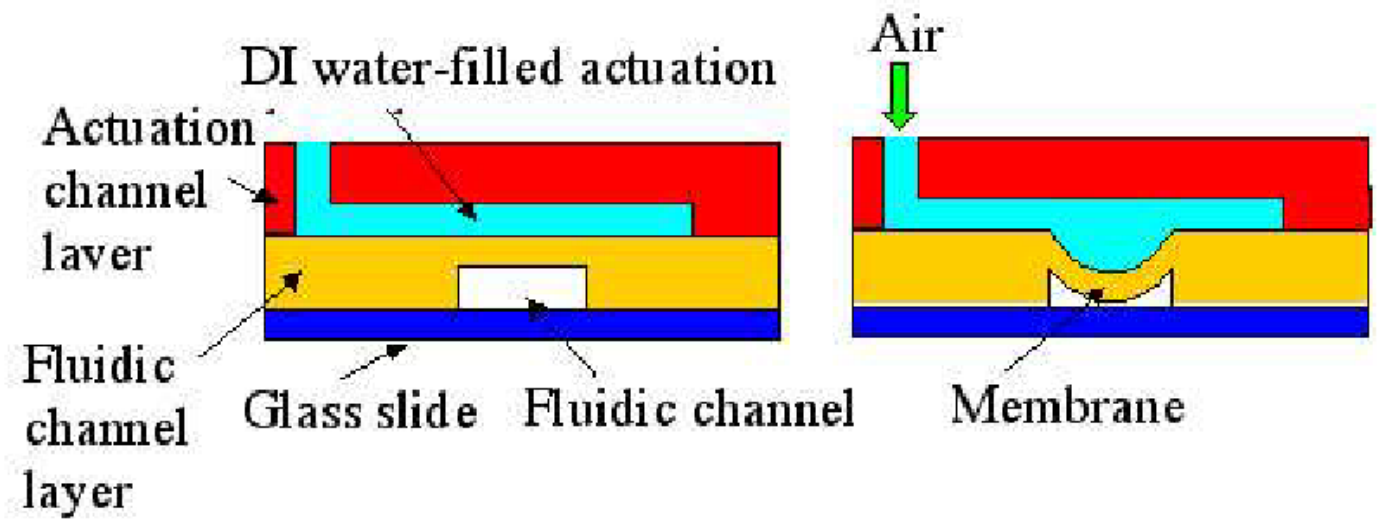

Fig. 2. PDMS valve actuation. Left: flow channel open; right: working channel closed. 


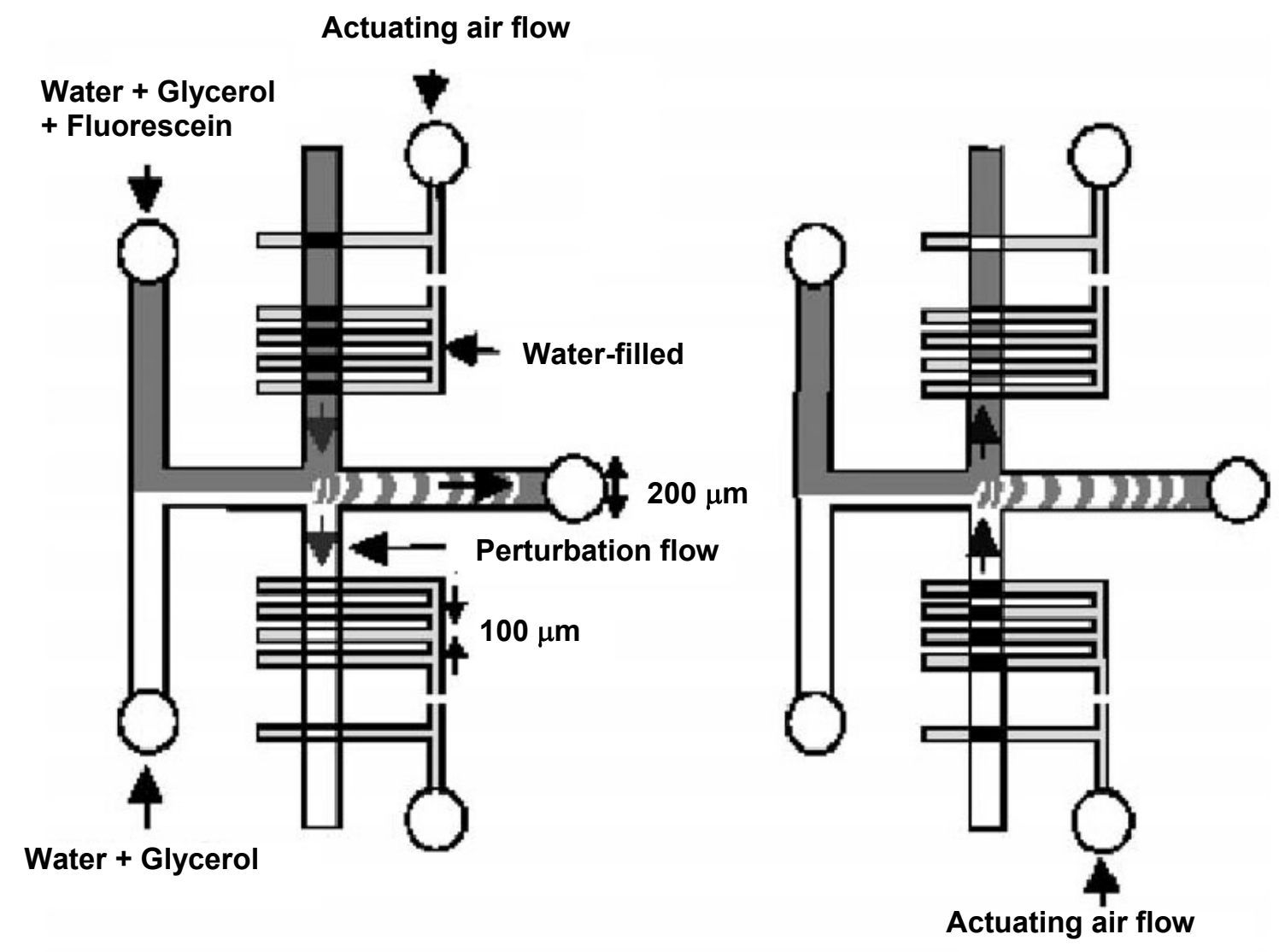

Fig. 3. Layout of the experimental system described in this article, with the operation mode. Left figure: upper valves closed and lower ones open, producing a side flow oriented downwards. Right figure: opposite movement, producing an upwards side flow. 
A. Dodge et al., C.R. Physique 5 (2004), 557-563.

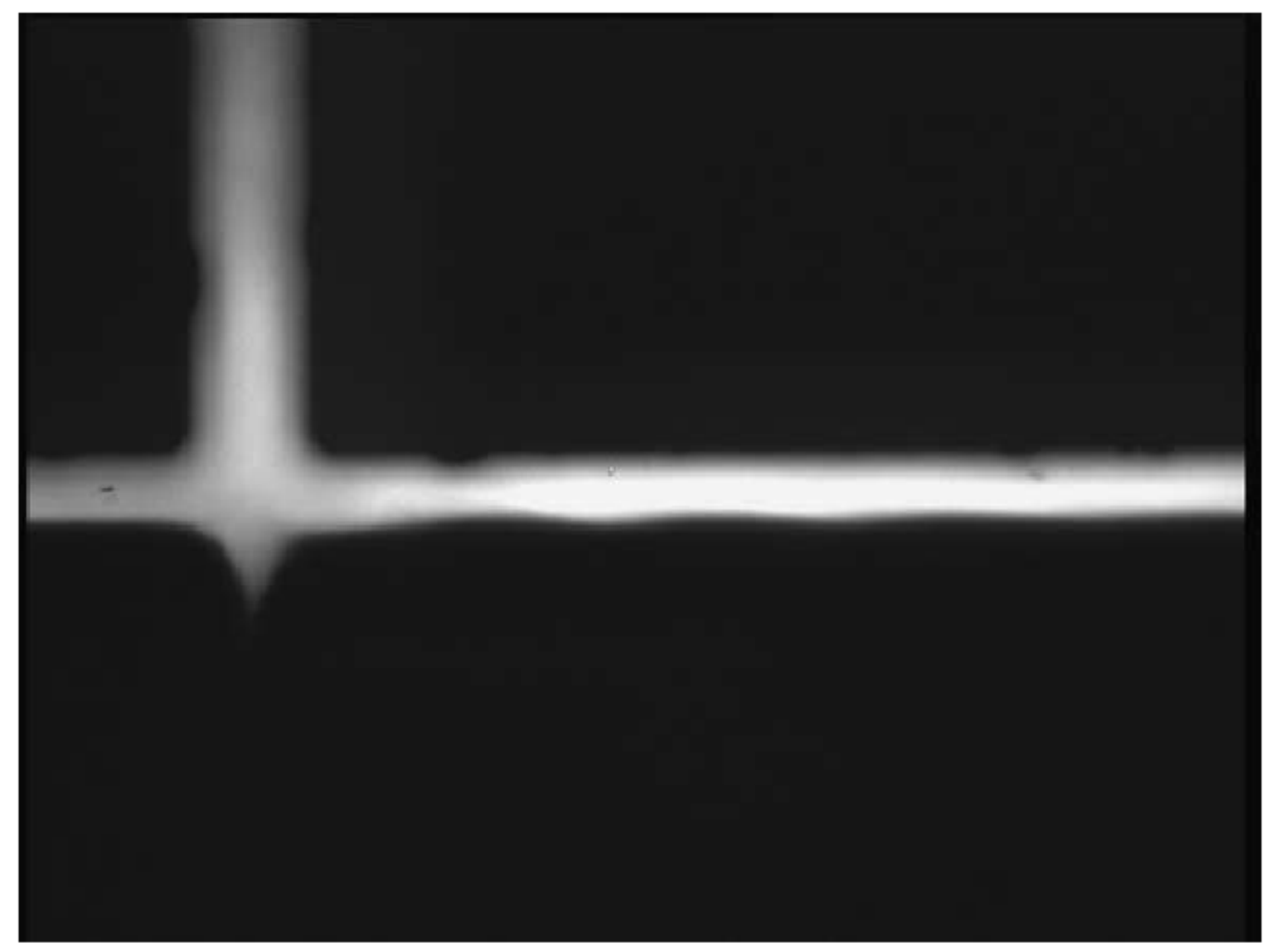

Fig. 4. Flow in the cross-channel micromixer, visualized from above. The fluids are mixtures of fluorescein and water. One fluid is labeled with fluorescein, the other not. In this particular case, the amplitude of the oscillatory pressure, applied on the membranes (see Section 2.3) is 0.4 bars, and the frequency is $1 \mathrm{~Hz}$. 
A. Dodge et al., C.R. Physique 5 (2004), 557-563.

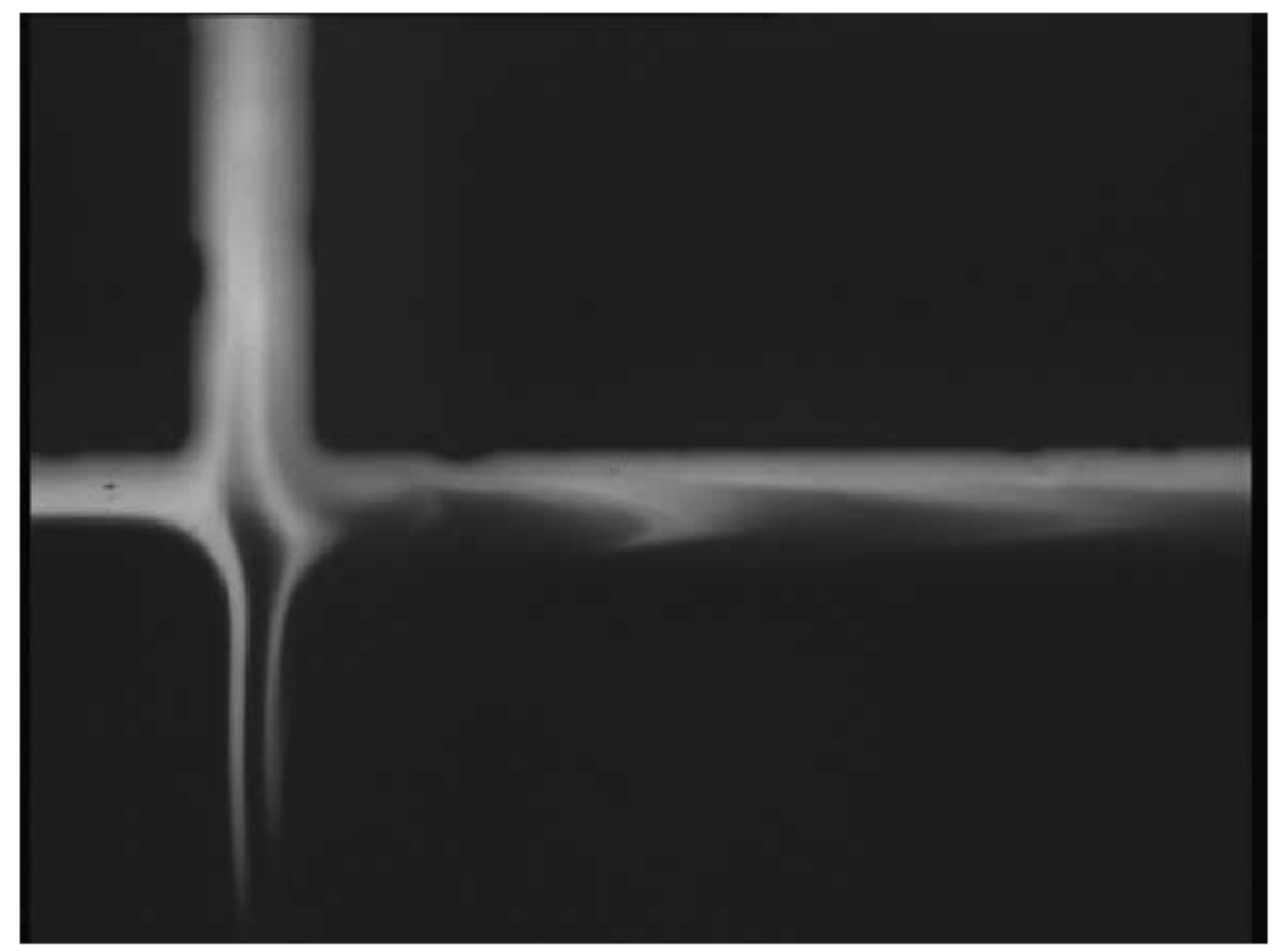

Fig. 5. Same conditions as Fig. 4, but with an amplitude of 1.45 bars and a frequency equal to $0.6 \mathrm{~Hz}$. 
A. Dodge et al., C.R. Physique 5 (2004), 557-563.

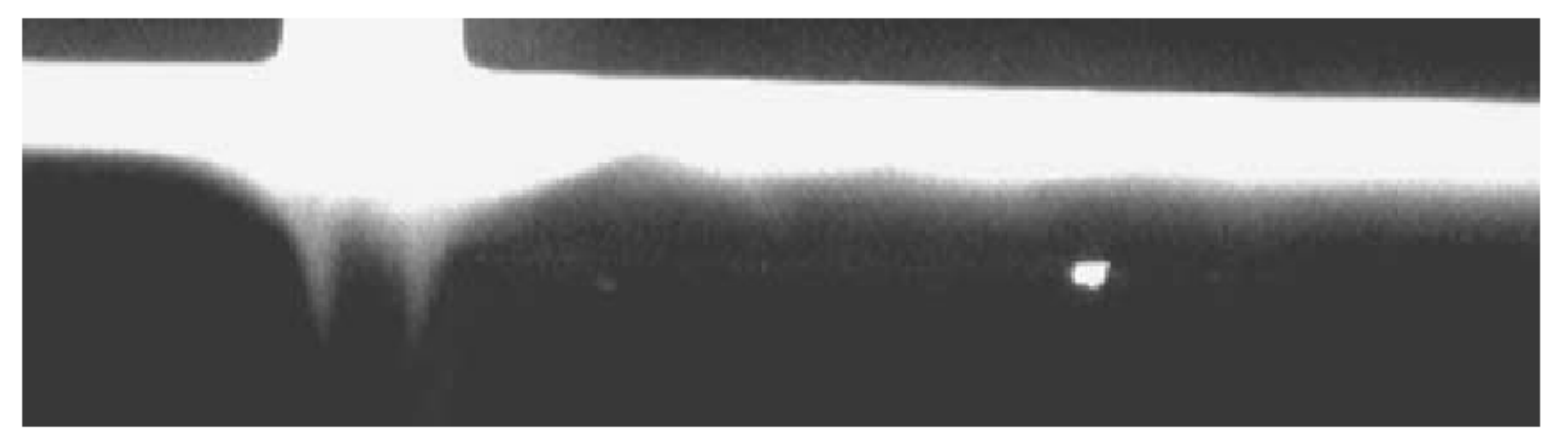

Fig. 6. Typical pattern in the resonance observed in the experiment. 
A. Dodge et al., C.R. Physique 5 (2004), 557-563.

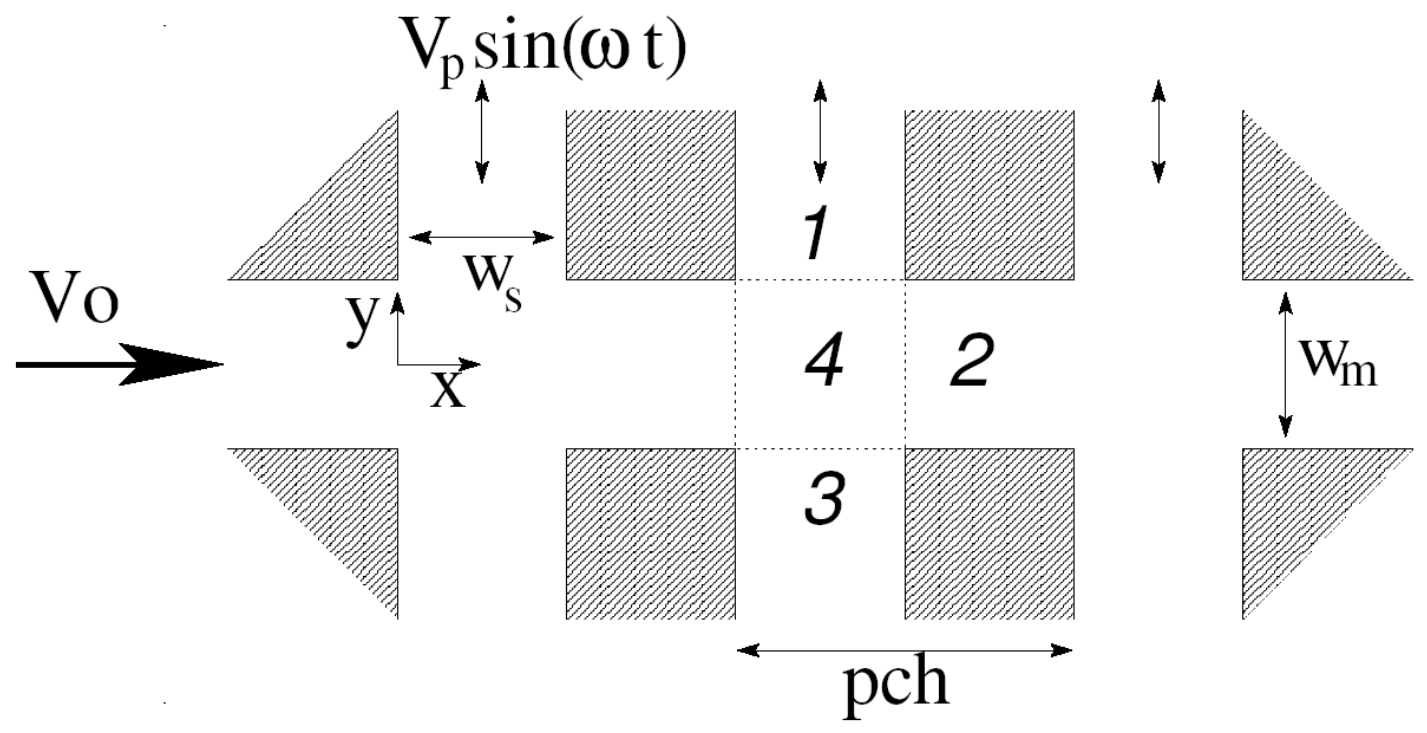

Fig. 7. Schematic showing the Lee mixer with multiple side channels. 

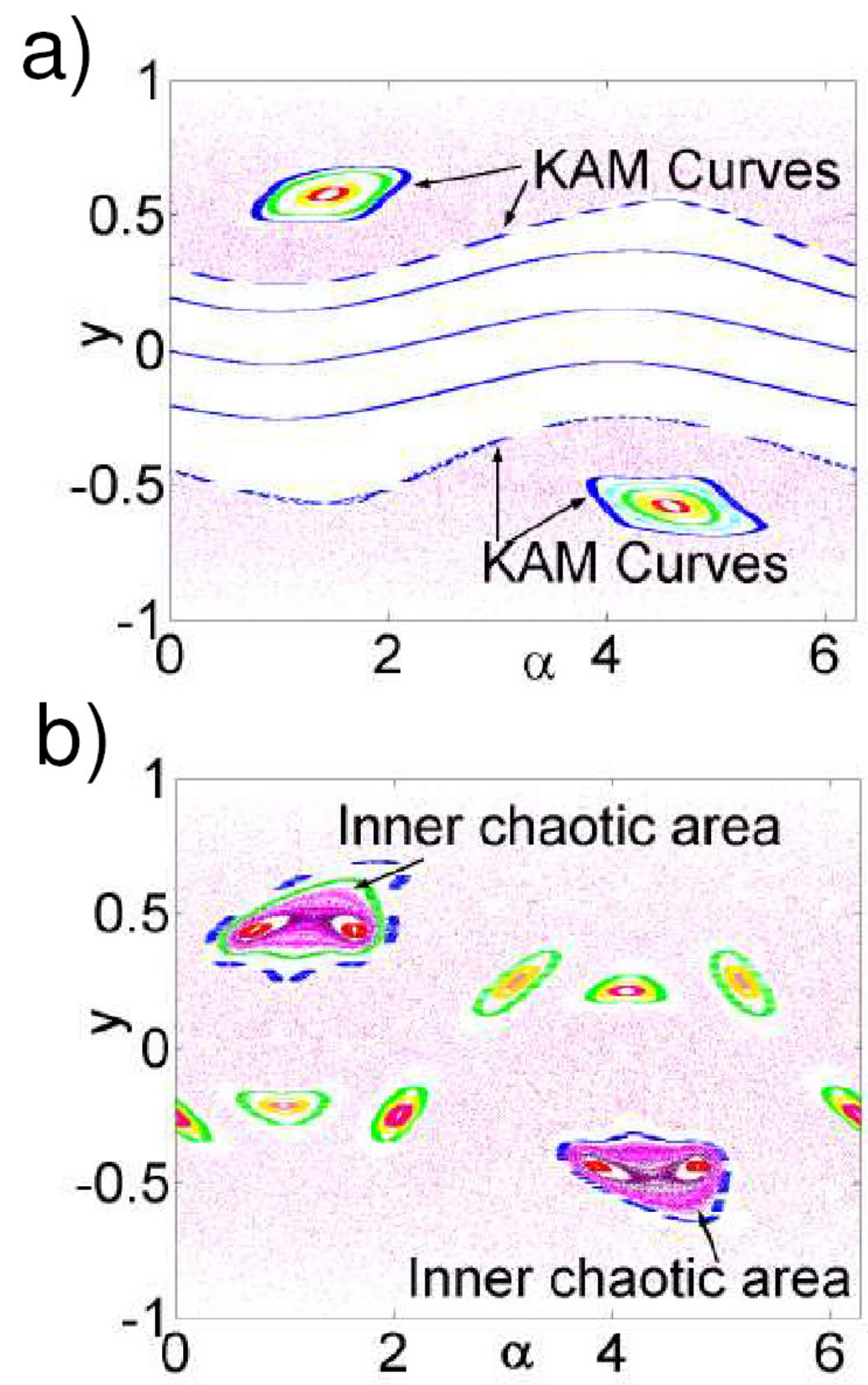

Fig. 8. Poincaré sections of the chaotic mixer for different values of $v_{p}$ : (a) 0.15 ; and (b) 0.43 . 
A. Dodge et al., C.R. Physique 5 (2004), 557-563.

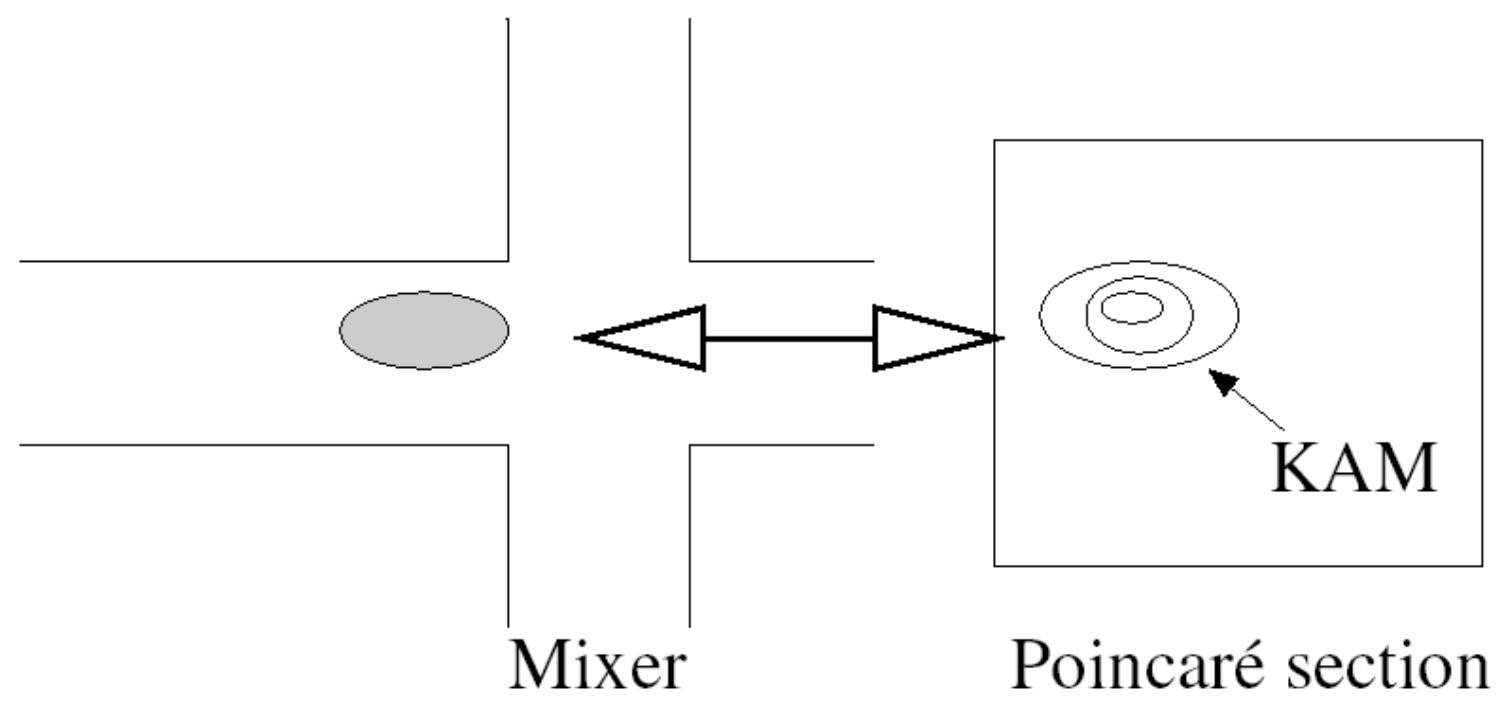

Fig. 9. Schematic showing the mapping between KAM curve in the Poincaré section, in the particular case of a quasi-periodic motion. 